\title{
Acercamiento empírico a las equivalencias de los verbos de desplazamiento en español y alemán ${ }^{1}$
}

\author{
JUAN CUARTERO OTAL \\ Universidad Pablo de Olavide \\ jcuartero@upo.es
}

\begin{abstract}
Resumen
La lexicografía bilingüe tiene dificultades para tratar los verbos de desplazamiento del alemán y el español. Algunos autores relacionan esas dificultades con diferentes patrones de lexicalización en ambas lenguas, no obstante, es posible mostrar que las dificultades se relacionan especialmente con las diferentes propiedades gramaticales de los verbos, las diferentes preferencias de uso de los hablantes y los diferentes elementos léxicos disponibles. Este trabajo ofrece una perspectiva general de esos fenómenos y un planteamiento contrastado de las posibilidades de presentación bilingüe de los verbos de desplazamiento de estas lenguas a partir de Gramática contrastiva, Lexicografía y Lingüística de corpus.
\end{abstract}

\section{Palabras clave}

Verbos de desplazamiento, gramática contrastiva, lexicografía bilingüe, corpus lingüísticos.

\footnotetext{
${ }^{1}$ El presente trabajo se ha llevado a cabo en el marco del proyecto COMBIDIGILEX (MINECO-FEDER FFI-2015-64476-P).
} 


\title{
Empirical approach to the equivalences between motion verbs of German and Spanish
}

\begin{abstract}
With bilingual lexicography, there are some difficulties in dealing with motion verbs of German and Spanish. Some authors relate these difficulties to different lexicalization patterns in both languages. However, it is possible to show that these difficulties are specially related to different grammatical properties of motion verbs, to different usage preferences of speakers and to different lexical elements available in each language. This paper deals with a general perspective of these phenomena and an approach to the possibilities of bilingual lexicographic presentation of motion verbs on the basis of Contrastive Grammar, Lexicography and Corpus Linguistics.
\end{abstract}

\section{Keywords}

Motion verbs, Contrastive Grammar, bilingual lexicography, corpus linguistics.

Recibido el 03/07/2019

Aceptado el 01/12/2019 


\section{Objetivos y marco de trabajo}

En los diccionarios bilingües del par español-alemán podemos hallar equivalencias para verbos de desplazamiento (en adelante, VVD) en una y en otra dirección que no resisten un acercamiento hecho desde la Gramática Contrastiva y con datos de corpus paralelos. Varios trabajos previos (Cuartero 2016 y 2017a) insisten en las dificultades que plantean las correspondencias que se establecen entre VVD del español y del alemán. Ambos trabajos parten de una base previa (Cuartero 2015 y 2017b) para analizar la representación lexicográfica en diccionarios bilingües de VVD concretos. Este trabajo completa dos aspectos pendientes: a) comprobar esas observaciones y conclusiones con datos empíricos por un lado de equivalencias de VVD en diccionarios bilingües y por otro de traducciones de descripción de eventos de desplazamiento en corpus paralelos y b) contrastar ambas informaciones para comprobar el alcance y validez de las dificultades teóricas previstas.

Por cuestiones de espacio el trabajo se centra en ejemplos escogidos español-alemán / alemán-español obtenidos de dos corpus paralelos de referencia creados ad hoc: el primero incluye las 1192 expresiones de desplazamiento halladas en la novela La piel del tambor (1995) de A. Pérez Reverte (en adelante, PT) y sus traducciones al alemán, que están en la versión Jagd auf Matutin (1997) de C. Schmitt (en adelante JM). El segundo incluye las 1315 expresiones de desplazamiento halladas en la novela Die unendliche Geschichte (1979) de M. Ende (en adelante UG) y sus traducciones al español, que se hallan en La historia interminable (1991), de M. Sáenz (en adelante $\mathrm{HI}$ ).

El análisis se centra en diccionarios electrónicos, que son los más usados en la actualidad y en los que se deben centrar los desarrollos teórico-prácticos venideros ${ }^{2}$ : se han consultado los dos más conocidos y empleados, Pons y Langenscheidt (en las citas se marcarán en adelante como PON y LAN); otro muy popular por la cantidad de equivalencias que ofrece, Leo (en las citas, en adelante, LEO) y otros dos menos ambiciosos, Beolingus y dict.cc (en adelante, BEO y DIC), que permiten contrastar las observaciones hechas en los demás.

\footnotetext{
2 Un trabajo de Domínguez (2016a: 290) indica que los diccionarios electrónicos se han impuesto a los impresos: su UDALPE indica que el 75 \% de los usuarios recurren a diccionarios electrónicos; su Ü-encuesta es aún más tajante, ya que indica que los emplea el 93,24\%.
} 


\section{Los WVD en diccionarios bilingües de alemán y español}

\subsection{Caracterización de los VVD en español y alemán}

Por un lado, el procedimiento de expresión de desplazamiento en ambas lenguas parece, en líneas generales, muy similar: ambas establecen combinaciones entre una serie de elementos comunes disponibles, a) un núcleo predicativo, b) un sujeto, c) una o varias indicaciones locativas (complementos preposicionales de equivalencia adverbial, adverbios o, con menor frecuencia, un objeto directo locativo) y d) complementos modales (gerundios, participios o adjetivos en función predicativa, sintagmas preposicionales o adverbios).

Por otro lado, y de acuerdo con la bien conocida propuesta de Talmy (2000), el alemán y el español pertenecen a dos tipos de lenguas distintas, las de marco satelital (satellite-framed) y las de marco verbal (verb-framed), que se reconocen en los distintos mecanismos predominantes en la lexicalización de los VVD.

Entre uno y otro punto de vista, el español (y las demás románicas) por un lado y el alemán (y las demás germánicas) por otro, aunque próximas en lo que respecta a lo geográfico y lo tipológico, presentan diferencias en su forma de describir eventos de desplazamiento (Cuartero 2015 y 2016). Los aspectos más reseñables que se observan en el contraste entre los VVD de ambas lenguas son:

1) Diferente sintaxis: muchos VVD del español muestran restricciones combinatorias con las indicaciones locativas dinámicas (*Vamos de la casa o *Corren del parque) mientras que los del alemán se combinan sin apenas restricciones (Sie gingen aus dem Haus o Sie sind aus dem Park gerannt). Una excepción son los VVD transitivos de ambas, que siempre tienen restricciones, pues el CD indica un punto de referencia local (Cuartero 2016): Cruzan la frontera (pero *Alcanzan el parque hacia casa) o Sie betreten das Haus (pero *Sie betreten das Haus in die Küche).

2) Diferentes tendencias de lexicalización: en español, los VVD lexicalizan de modo saliente o Trayectoria (venir, volver etc - en adelante VVDT) o Manera (correr, caminar etc- en adelante VVDM) ${ }^{3}$; en alemán, los VVD lexicalizan Manera

\footnotetext{
${ }^{3}$ Aunque algunos han lexicalizado ambos componentes (escapar, retroceder, zarpar) y otros no parecen lexicalizar ninguno (trasladarse, circular).
} 
(fahren, eilen, laufen etc) y solo muy pocos, Trayectoria (gehen (en la acepción de 'irse'), kommen, gelangen etc).

3) Diferentes preferencias de uso: en español, los VVD más usuales lexicalizan la Trayectoria (ir, venir, salir, entrar, llegar, volver y pasar $)^{4}$. En alemán, hay unas pocas piezas léxicas muy frecuentes (kommen y gehen, y sus variantes con partículas o prefijos) ${ }^{5}$, y gran dispersión en el uso de otros VVD.

Una dificultad añadida en relación con este último aspecto es que la Lexicografía del alemán considera los VVD compuestos mediante partículas como una unidad léxica, y así aparecen (o podrían aparecer) en la macroestructura de los diccionarios: como lemas se incluyen partículas (herein-, heraus-, etc.) y compuestos frecuentes (hereinkommen, herausfahren, etc.). Autores como Engel (1996: 442), Wotjak (1997: 312) y Engelen (2010: 57s), opinan, no obstante, que no deberían considerarse verdaderos compuestos: esas partículas no son prefijos sino indicaciones adverbiales que solo se escriben unidas al verbo por convención ortográfica.

\subsection{Los diccionarios bilingües}

Domínguez (2016b: 171ss) describe el comienzo de una nueva era en la Lexicografía: tenemos nuevos medios, nuevos tipos de diccionarios, atención a las necesidades de cada tipo de usuario y usuarios activos, sea como redactores (en Pons o Leo), sea como creadores de diccionarios (en Wiktionary).

Los nuevos diccionarios electrónicos permiten superar las limitaciones de los diccionarios bilingües «convencionales»: espacio disponible para cada entrada, posibilidades de revisión y puesta al día, y lo que podría llamarse «tradición lexicográfica». Los diccionarios en línea presentan evidentes ventajas en los dos primeros aspectos; con esa «tradición» de los diccionarios bilingües, se atiende implícitamente a la denominada «ley de sinonimia» (Seco, 1987: 36), que a una palabra tiende a hacerle corresponder en lo posible otras de la misma categoría gramatical, monolexemáticas o al menos con forma escueta. Así, estas equivalencias se establecen

\footnotetext{
${ }^{4}$ Datos tomados de la Base de Datos Sintácticos (BDS), buscando el esquema [Sujeto + Complemento adverbial]. Las cifras de aparición son: ir (791 registros); salir (572); llegar (523); entrar (473); venir (310); volver (308) y pasar (287).

${ }^{5} \mathrm{~A}$ lo largo de este trabajo me permito referirme de modo genérico a las formas de un VD compuestas por adición de partículas marcando ese uso mediante un guion previo (-kommen, -gehen, etc).
} 
conformando un diccionario de sinónimos en dos lenguas distintas (Baldinger 1971). De hecho, tal y como los diccionarios bilingües están concebidos y redactados, sirven para la traducción y la comprensión de textos, pero no tanto para su producción (Haensch et al. 1982: 285ss). En ellos se esperan y se prefieren unidades léxicas funcionalmente equivalentes, que reproduzcan en la lengua meta el significado de la entrada y puedan aparecer en diversos contextos. Lo normal es que esas correspondencias sean traducciones y que las definiciones o paráfrasis solo se den cuando no haya otra posibilidad (como con referencias culturales o realia). Esa es aún una asignatura pendiente de los diccionarios electrónicos, si se consideran diversas circunstancias que trata en detalle Meliss (2016: 194ss): buena parte de los diccionarios bilingües en línea consisten primordialmente en volcar datos preexistentes de un diccionario en papel, mientras que los diccionarios de nueva planta y los colaborativos evidentemente intentan asimilarse a las obras previas.

\subsection{Dificultades para la representación de VVD}

Fuentes (1997: 2) afirma en un escueto pero severo juicio que «no es frecuente que se elaboren diccionarios bilingües con perspectivas científicas». Si consideramos la tendencia a dar equivalencias sinonímicas y no definiciones ni paráfrasis, resultan un problema los paralelos léxicos forzados y equívocos que a veces contienen los diccionarios, manuales y glosarios con VVD de estas lenguas (sin necesidad de ir más lejos, parejas como ir-gehen o venir-kommen). Como advertía Hess (2007: 291-293) en su valoración del tratamiento de los VVD en diccionarios español-alemán, los resultados siempre dejan mucho que desear.

Las diferencias que hay en lo léxico, lo sintáctico y lo semántico son un problema para encontrar equivalencias léxicas entre VVD, y tienen al menos tres consecuencias directas relacionadas con la representación lexicográfica de los VVD:

1) Los VVDT españoles (p. ej. salir o volver) suelen recibir como equivalencias en alemán VVDM compuestos (herauskommen, -gehen etc o zurückkehren, -kommen, -gehen etc).

2) Los VVDM alemanes con elementos que indican Trayectoria (p. ej. zurückgehen o hinauffahren) no siempre tienen equivalencias españolas simples (regresar caminando) o claras ( $\approx$ subir montado en un medio de transporte). En general, se 
recurre a correspondencias que prescinden de la indicación de Manera (volver o subir, respectivamente).

3) Los VVDM (p. ej. laufen, fliegen o andar, volar) suelen tener equivalentes en ambas lenguas con propiedades combinatorias muy distintas.

Por todo ello, las equivalencias frecuentes resultan ser más una opción de traducción ad hoc que una correspondencia que dé cuenta del significado del lema: unas veces son un hipónimo que contiene más información que el lema, pero permite la sustitución directa (como vemos en 1), otras veces son un hiperónimo que contiene menos información que el lema, pero permite la sustitución directa (como en 2), finalmente, otras veces son un sinónimo pero que no permite traducir al otro en todos los casos (como en 3). Resulta interesante observar que las correspondencias tratan de indicar cómo expresar en ciertos casos el significado de una palabra de la L1 en la L2 (la información que ofrecen los diccionarios) y no tanto cómo se puede expresar (la información que ofrecen las gramáticas) y mucho menos cómo se suele expresar (la información que ofrecen los corpus).

\section{Dificultades en el tratamiento lexicográfico de los VVD}

\subsection{Equivalencias de VVDT españoles}

Los VVD más frecuentes en español (ir / venir, salir / entrar, llegar, volver y pasar), no siempre encuentran una equivalencia exacta en alemán: suele hacerse con un VD que a su vez puede contener la información sobre Manera (que no hay en el lexema español) y con alguna partícula que determina fuertemente el paralelismo léxico. Como muestra se van a exponer y comentar datos extraídos de ambos corpus con las correspondencias de expresiones con volver / regresar: en un caso las expresiones que se emplean para traducirlos al alemán en JM y en otro las expresiones del alemán de HI que se tradujeron mediante alguno de los dos.

\subsubsection{Datos de los corpus}

Correspondencias entre PT y JM (español-alemán):

1) Traducciones de volver: 17 token, de los cuales 16 se han traducido mediante un VD alemán. Los 16 ejemplos se corresponden con 8 VVD diferentes: los dos más frecuentes son zurückkommen (5 veces) y zurückkehren (4). También hay varias 
«expresiones equivalentes que no necesariamente expresan Manera»: wieder klettern, wieder besuchen, kehrtmachen y nochmals hierher kommen.

2) Traducciones de regresar: 20 token, de los cuales 16 se han traducido mediante VVD. Los 16 ejemplos se corresponden con 9 VVD diferentes: los más frecuentes son zurückkommen (4) y zurückkehren (4). Las otras expresiones son zurückgehen (2), heimgehen (1) y wieder erscheinen (1) (las tres únicas que expresan Manera) así como wieder präsent sein, heimkehren, wieder erscheinen y wieder zurück mögen.

3) En total, para los 32 token considerados, se han empleado como correspondencias en alemán 12 VVD diferentes: los más llamativamente frecuentes son zurückkommen (9 - 30 \%) y zurückkehren (8 - $25 \%$ ), que juntos son poco más de la mitad ( $56 \%$ ) de todos los casos. Las formas más reiteradas son: zurück- (20 - 62,5\%); -kehren (13 - $40 \%)$; -kommen (9 - $28 \%)$ y wieder$(7-22 \%)$.

Las correspondencias entre UG y HI (alemán-español):

1) Traducciones con volver: 62 token, que se corresponden con 17 expresiones diferentes: las dos más frecuentes son zurückkehren (24 veces) y zurückkommen (10), que no indican Manera. También hay varias expresiones repetidas: umkehren (6), zurückgehen (4) wiederkommen (4), zurückwollen (2) y zurückmüssen (2). Las demás aparecen una vez: zurückhuschen, zurücklaufen, zurückwatscheln, zurückkönnen, zurückziehen, heimkehren y otras como nach Hause gehen y nach Hause können, zu jdm fliegen y solamente zurück.

2) Traducciones con regresar: 14 token que se corresponden con 7 expresiones diferentes. La más frecuente es zurückkehren (8 - 57 \%), las otras seis aparecen solo una vez: heimkehren, umkehren, zurückgehen, zurückfliegen, zurückwollen y zurückkönnen.

3) En total, para los 76 token considerados, se han empleado 18 formas diferentes como correspondencia en alemán: las más llamativamente representativas son zurückkehren (32 - $42 \%$ ) y en menor medida zurückkommen (10 - $13 \%$ ), que juntas suponen más de la mitad (55,2 \%) de todos los casos. Las formas de expresión más reiteradas son, por otro lado: zurück- (48 - $63 \%$ ); -kehren $(30-39,5 \%)$ y -kommen $(14-18 \%)$. 


\subsubsection{Análisis}

De los datos obtenidos de ambos corpus se puede observar que a pesar de las diferencias en el origen y en el número de token considerados se producen diversos paralelismos evidentes:

- Más de la mitad (55\%) de las equivalencias alemanas en ambas direcciones son dos VVD compuestos, zurückkehren y zurückkommen, que no conllevan indicaciones de Manera. En el corpus del DWDS, las formas más usuales son zurückkehren (3633 registros frente a wiederkehren con solo 637), zurückkommen (1991) у zurückgehen (1915).

- La aparición de volver y regresar se asocia muy fuertemente con la presencia de la partícula zurück- (62\%) y de un verbo concreto, -kehren (39\%) y en menor medida, -kommen (23-21,2\%).

- Hay fuerte dispersión en los usos del alemán: dos VVD compuestos muy frecuentes y unos pocos de frecuencia baja: zurückgehen (5), wiederkommen (4) y zurück- con los verbos modales, -wollen (3), -müssen (2) y -können (2). Los 11 casos restantes aparecen una sola vez.

- Hay un elemento de contraste llamativo: entre las equivalencias traducidas del español aparecen diversos VVDM (5 de entre 15 expresiones - 6 ejemplos de 32: $18,8 \%$ ) pero en el texto original alemán aparecen incluso menos (6 de un total de 18 expresiones - 10 ejemplos de 76: 13,1\%).

\subsubsection{Tratamiento en diccionarios bilingües}

Los datos que nos muestran los diccionarios son los siguientes:

volver: zurückkehren nach +dat/aus/von +dat / ( a casa) heimkehren (PON); zurückkehren / zurückkommen / [...] umkehren / wiederkehren / wiederkommen / zurückfahren / (volando) zurückfliegen / zurückgehen / zurücklaufen [...] (LEO); ( $\approx$ regresar) zurückkommen, -kehren / (volver en vehículo) zurückfahren (LAN); zurückkommen / zurückkehren (DIC); [...] wiederkommen / [...] umkehren / [...] zurückkommen / [...] wiederkehren (BEO).

regresar: zurückkehren (PON); zurückkehren / ( $a$ casa) heimkehren / zurückkommen / ( $a$ casa) heimreisen (LEO); zurückkehren (LAN); zurückkommen / (a un lugar lejano) zurückkehren (DIC); zurückkommen / [...] wieder da sein / zurückkehren / [...] umkehren [...] (BEO).

Hay varios hechos que llaman especialmente la atención: 
- Siendo dos VVD sinónimos, volver tiene en todos los diccionarios más correspondencias que regresar (en total 20 frente a 12).

- Ninguno de los diccionarios da las mismas equivalencias para volver y regresar.

- Aparecen algunas equivalencias poco representativas (heimreisen o wieder da sein) y faltan otras muy frecuentes (zurückgehen).

\subsubsection{Contrastes}

De los datos observados en los diccionarios, es claro que el peso semántico de las correspondencias recae en la partícula zurück- (20 veces) y en el verbo -kehren (17), también en cierta medida, wieder- (5) y -kommen (9); eso mismo se desprende de los datos del corpus. Considerando esos datos, los diccionarios ofrecen equivalencias adecuadas si se trata de recepción, pero no tanto si se trata de producción. Se puede indicar lo siguiente:

- Para ambos VVD, la forma zurückkehren es la equivalencia más frecuente en los diccionarios (solo falta en Beolingus para regresar) junto con zurückkommen (que no aparece en las entradas de Pons).

- Solo Leo da muestras de que zurück- combinada con VVD produce equivalentes de volver / regresar, Langenscheidt ofrece un único ejemplo.

- La partícula wieder-, que solo aparece con kehren y kommen, no suele estar entre las equivalencias: aparte de wieder da sein, solo está en dos diccionarios, dos veces en la misma entrada pero solo con volver.

- La partícula heim- apenas aparece (3 veces en total) y lo hace muy heterogéneamente: Leo la incluye dos veces, pero solo para regresar y Pons, solo una en volver.

Como conclusión, en estos casos se echa de menos sistematicidad. La información de los diccionarios en ambas entradas son algunos verbos reunidos, en general, sin un criterio evidente y de modo no exhaustivo: siempre aparecen una o las dos equivalencias más frecuentes según los corpus, pero las demás son altamente heterogéneas. No se indica ningún dato sobre gramática o combinatoria que permita ayudar a formar VVD compuestos, de por sí muy usuales pero, como se ha visto en los corpus, de uso disperso; si se hace es de modo implícito, y solo en la entrada volver. 


\subsection{Equivalencias de VVDT del alemán con o sin Manera}

Los VVD del alemán suelen expresar Trayectoria mediante una partícula adverbial, un sintagma preposicional o ambos. Si además expresan Manera, no suelen tener equivalencia léxica exacta en español. En este apartado se muestran y comentan datos extraídos de varias búsquedas cruzadas en el corpus: por un lado, las equivalencias de las construcciones con el verbo salir de PT y las que en JM contienen heraus-/hinauscon VVD y, por otro lado, las traducciones al español de las expresiones de UG que llevan las partículas heraus-/hinaus- y VVD, y las expresiones que se tradujeron mediante salir en HI.

\subsubsection{Datos de los corpus}

Las correspondencias entre PT y JM (español-alemán):

1) Traducciones de salir: 59 token, de los que solo 45 se han traducido mediante 12 VVD con distintas partículas y complementos. Los dos más frecuentes son verlassen y -treten (12 veces cada uno - $53 \%$ de los casos) mientras que -kommen (6) y -gehen (5) están relativamente menos representados: (33,3\%). El $14 \%$ restante son VVDM: -dringen, durchqueren, -fahren, -geleiten, -holen, -stechen, -steigen y -verkehren. No aparecen con mucha frecuencia las partículas heraus- (3) y hinaus- (8). El complemento direccional más frecuente es aus + Dat (11 - $25 \%$ ), que nunca aparece con partículas.

2) Traducciones con heraus-/hinaus- + VVD: no son especialmente representativas. Solo hay 16 token, de los cuales 8 son hinaustreten y uno, heraustreten (55\%). Asimismo, hinausgehen está dos veces y hinauskommen, una. El resto de los VVD son -folgen y -laufen así como -wollen y -wünschen, una vez cada uno. Por otro lado, solo los ejemplos con hinaustreten, heraustreten, hinausgehen y hinauskommen corresponden a expresiones con salir: los cuatro restantes se usaron para traducir otros VV.

Las correspondencias entre UG y HI (alemán-español):

1) Traducciones con salir: 76 token $^{6}$, que corresponden a 18 VVD diferentes con distintas partículas y complementos. Los más frecuentes son -kommen (18),

\footnotetext{
${ }^{6}$ Se han obviado todos aquellos ejemplos de salir en los que el significado es 'partir', 'amanecer' o 'aparecer'.
} 
-gehen (17) y -treten (10), que suponen el 59 \% de los casos. Asimismo, -steigen (6) y el transitivo verlassen (6) son dos VVD comparativamente frecuentes. El resto son o bien los modales -können (2), -müssen (2) y -mögen o bien VVDM: -arbeiten, -finden,- kriechen, -laufen, -poltern, -sprengen, -springen, -stürmen, -ziehen y -zischen. Las dos partículas más frecuentes son como acabamos de ver heraus- (9) y hinaus- (25) y el complemento direccional más frecuente es aus + Dat (31 - $40 \%$ ) y 19 de estos (25 \%) no llevan ninguna partícula.

2) Traducciones de heraus- / hinaus- + VVD: 45 token que contienen 17 VVD con clara preeminencia de -gehen (14), -kommen (8) y -treten (5), que juntos ya suponen el $60 \%$ de todos los casos. Los restantes VVD aparecen como mucho dos veces (-müssen, -kriechen, -finden y -laufen) y por lo general solo una (-arbeiten, -fahren, -huschen, -mögen, -tasten, -können, -stürzen, -begleiten, -treiben y -steigen). De los 45 ejemplos, 31 se tradujeron sin más como salir (66 \%), 10 mediante diversos VVD (dirigirse (4), cruzar (2), entrar, ir, acompañar y moverse) y solo 4 con VVDM (arrastrarse afuera, deslizarse, precipitarse y correr).

\subsubsection{Análisis}

Al revisar los datos obtenidos de los corpus, es interesante observar varias analogías claras:

- Se observa que tres VVD, -gehen (38 casos), -treten (36) y-kommen (33), son los más frecuentes en los cuatro casos revisados y que suponen siempre algo más de la mitad de los ejemplos en cada uno de ellos. El otro verbo frecuente en los datos hallados es verlassen (18). El resto de los VVD alemanes aparece de modo esporádico.

- Entre los ejemplos traducidos al alemán esta vez se encuentran muchos VVDM: 11 de un total de 16 y 38 ejemplos de 61 (57\%), de ellos 28 con-treten (21) y -gehen (7). Lo mismo ocurre en el texto original alemán: 19 de un total de 24 y 75 ejemplos de 121 (62\%), de los cuales 46 son -gehen (31) o -treten (15).

- En el texto original alemán es frecuente que las informaciones de Trayectoria sean aportadas por partículas (45\% de los casos) o por complementos Origen (40\%). En el $25 \%$ de los casos no necesitan de Trayectoria explícita. En el texto 
traducido del español, sin embargo, aparecen menos partículas, pero más VVDM con complementos locativos.

\subsubsection{Tratamiento en diccionarios bilingües}

Todos los diccionarios bilingües consultados consideran heraus- / hinaus- como adverbios:

heraus: ADV (hacia) fuera, afuera (PON); ADV afuera, fuera (LEO); ADV fuera, afuera (LAN); ADV fuera (DIC); lejos, fuera (BEO).

hinaus: ADV fuera, afuera (PON); ADV afuera, fuera (LEO); ADV (hacia) afuera, fuera (de aquí) (LAN); ADV afuera (DIC); ADV afuera, salir fuera [sic] (BEO).

Aunque así resulte más difícil entender y enseñar su significado, como también asimilar su empleo, todos los hacen corresponder con fuera y afuera, con las únicas excepciones de (hacia) fuera (PON), (hacia) afuera (LAN) y de lejos y el sorprendente salir fuera (BEO). Solo en Leo se dan las mismas equivalencias en ambas entradas.

El tratamiento de las entradas compuestas por VVD con las partículas hinaus- / heraus- es llamativamente desigual en los cinco diccionarios que se han revisado:

herausgehen: salir (PON); salir de algo, salirse de algo (LEO); salir (LAN); falta en DIC y BEO / hinausgehen: salir (PON); salir (LEO); salir (de) (LAN); salir (a pie) (DIC); salir (BEO).

heraustreten: salir de (PON); salir de algo (LEO); salir(se) (de) (LAN); falta en DIC y BEO. / hinaustreten: salir afuera (PON); falta en los demás.

herauskommen: salir (PON); salir de algo (LEO); salir (de) (LAN); salir, emerger (BEO); falta en DIC. / hinauskommen: salir (PON); salir (LEO); salir afuera, salir (LAN); falta en DIC y BEO. herausfahren: salir de (PON); salir de algo (LEO); salir (LAN); salir (en vehículo) (DIC); falta en BEO. / hinausfahren: salir (PON); pasear en coche, salir (LEO); salir (de) (LAN); salir, zarpar (BEO); falta en DIC.

herauslaufen: correr hacia afuera, salir corriendo de (PON); salir corriendo (LAN); correr hacia afuera (BEO); falta en DIC y LEO. / hinauslaufen: salir corriendo (PON); salir corriendo, ir a parar a (LEO); salir corriendo (LAN); salir corriendo, ir a parar en (BEO); falta en DIC.

herausfinden: saber salir de, encontrar la salida de (PON); saber salir de algo (LEO); encontrar la salida (BEO); falta en DIC y LAN. / hinausfinden: encontrar la salida (PON) encontrar la salida (LAN); falta en LEO, DIC y BEO.

herausmüssen: tener que salir (de) (PON); tener que salir (LAN); falta en LEO, DIC y BEO. / hinausmüssen: tener que salir (LAN); falta en los demás.

herauswollen: querer salir de (PON); querer salir (LAN); falta en LEO, DIC y BEO. / hinauswollen: querer salir (PON); querer salir (LAN); falta en LEO, DIC y BEO como VD. 
herausarbeiten: salir, abrirse paso, conseguir salir de algo (PON); salir de (LAN); falta en LEO, DIC y BEO. / hinausarbeiten, en ninguno.

herausstürzen: salir corriendo de, salir precipitadamente de (PON); salir precipitadamente (LAN); falta en LEO, DIC y BEO. / hinausstürzen: caer, salir precipitadamente, [refl.] tirarse (PON); salir precipitadamente, [refl.] tirarse (LAN); falta en LEO, DIC y BEO.

herauskriechen: salir arrastrándose (LAN); falta en los demás. / hinauskriechen: arrastrarse hacia afuera (LEO); falta en los demás.

herauskönnen: poder salir (LAN) falta en los demás. / hinauskönnen: poder salir (PON); falta en los demás.

herausbegleiten: acompañar hacia afuera (PON); falta en los demás. / hinausbegleiten: acompañar hacia fuera (PON); acompañar a alguien afuera (LEO); acompañar afuera, acompañar hasta la puerta (LAN); falta en DIC y BEO.

heraustreiben: expulsar (PON); falta en los demás diccionarios. / hinaustreiben: echar, sacar (PON); expulsar (LEO); falta en los demás.

heraussteigen: falta en todos los diccionarios. / hinaussteigen: salir afuera (PON); falta en los demás diccionarios.

heraus- e hinaushuschen, heraus- e hinaustasten, heraus- e hinausmögen, heraus- e hinauswünschen así como heraus- e hinausfolgen no aparecen en ninguno de los cinco.

Una rápida ojeada revela asimismo la poca homogeneidad del tratamiento en general de estas (posibles) entradas tanto desde el punto de vista de la macro como de la microestructura.

Algo parecido sucede con las equivalencias de verlassen mediante salir, que solo recogen tres de los cinco diccionarios:

verlassen: (hinausgehen, -fahren) dejar, salir de (PON); dejar, salir de (LAN); 23. salir de la casa (BEO); no aparece en LEO ni DIC con la equivalencia salir.

En total, de 200 posibles entradas con VVD compuestos, los diccionarios han incluido 64. Los datos están, no obstante, determinados por las enormes diferencias en el número de entradas que ofrecen los diccionarios: Pons y Langenscheidt son los que más, 24 y 20 respectivamente, Leo contiene 12, Beolingus 7 de ellas y dict.cc solamente 2. En esas 64 entradas, el verbo salir se nombra 56 veces, aunque la expresión salir afuera también está inexplicablemente tres. Por otro lado, no es frecuente que los diccionarios ofrezcan con rigor ambas formas compuestas.

A su vez, las equivalencias para salir que hallamos en la dirección español-alemán resultan bastante arbitrarias:

salir: hinausgehen, ausgehen (PON); (aus etw.dat) herausfahren, herauskommen, hervorkommen, hervorströmen, hinausgehen, hinauskommen, herausgehen, ausfahren 
(LEO); ausgehen, fort-, weggehen, herauskommen, hinausgehen (LAN); ausgehen [weggehen], hinausgehen, [...] herausfahren (DIC); hinausziehen (weggehen), hinausgehen, herauskommen, ausgehen, austreten, weggehen, hinausfahren (Zug) (BE0).

\subsubsection{Contrastes}

Del cruce de los datos de los corpus y de los diccionarios surgen una serie de observaciones:

- Las entradas con los VVD más representativos del corpus aparecen desigualmente en los diccionarios: hinausgehen es el único que aparece en todos y herausgehen, solo en tres; heraus- e hinauskommen aparecen en tres y cuatro respectivamente.

- En contraste, heraustreten solo aparece en tres diccionarios e hinaustreten, en uno, aunque son tan frecuentes en el corpus; ello contrasta con la presencia de heraus- e hinausfahren (cuatro veces cada uno) y de heraus- e hinauslaufen (tres y cuatro respectivamente).

- Las equivalencias que se prefieren en español suelen obviar las indicaciones sobre Manera de desplazamiento, muy claramente con -gehen, -treten y -laufen.

- Entre las equivalencias de salir resulta llamativo que el VD más frecuente sea -gehen (12 veces), y que -kommen (4) aparezca tantas veces como -fahren (4) mientras que -treten aparece una sola, y precisamente en Beolingus.

- Entre estas, se indica muy frecuentemente alguna Manera de desplazamiento -algunas incluso inesperadamente- (como weggehen, hinausziehen o hervorströmen).

La conclusión de nuevo es poco elogiosa en lo que respecta al tratamiento de los datos: las entradas demuestran, en general, cierta falta de criterio y de exhaustividad, añaden o eliminan matices de significado y los dobletes con hinaus- y heraus- a menudo no se recogen - y en caso de hacerlo no suelen contener la misma información.

\subsection{Correspondencias de VVDM que no indican Trayectoria}

En líneas generales, es cierto que los verbos que refieren el modo de desplazarse encuentran equivalencias léxicas relativamente sencillas en la otra lengua, que se suelen solucionar en la mayor parte de los casos de modo bastante satisfactorio mediante equivalencias monoléxicas. En Cuartero (2016) se discute, no obstante, que esas 
equivalencias «más fáciles» ocultan ciertas diferencias profundas relacionadas con la combinatoria de estos verbos que, como he dicho, no muestran restricciones en alemán pero sí muy frecuentemente en español. A ello se añade otra dificultad que se observa al hilo de los datos que se extraen del corpus acerca de las correspondencias de expresiones con los VVD caminar / andar.

\subsubsection{Datos de los corpus}

Las correspondencias entre PT y JM (español-alemán):

1) Traducciones de andar: 14 token, de los cuales todos se han traducido mediante un VD. Los ejemplos corresponden a 8 VVD diferentes: el más frecuente es -gehen (5 - $35 \%$ ), -schreiten y -treten aparecen dos veces cada uno, los otros cinco solo una: sich begeben, einbiegen, -spazieren, -aufstellen y -weichen. No hay partículas que aparezcan más de una vez, todas determinadas por el cotexto narrativo: vorbei-, vor-, zu-, entlang-, herum-, zurück-.

2) Traducciones de caminar: 42 token, de los cuales 40 se han traducido mediante VVD. Los ejemplos abarcan 15 expresiones diferentes, de las que tres VVD son más frecuentes: -gehen ( 9 - 22,5\%), -schreiten (7 - 17,5\%) y -schlendern (5-12,5 \%). Tres VVD aparecen dos veces (sich begeben, zurücklegen y folgen) y los nueve restantes, una sola (-kommen, überqueren, -eilen, -tappen, -wandern, verschwinden, -spazieren, bewegen, sich in Bewegung setzen). No hay partículas con frecuencia llamativa: aparece tres veces zuschreiten y dos hinabschlendern.

3) Para los 54 token considerados, hay 19 correspondencias en alemán: las más representativas son -gehen $(14-25 \%)$ y -schreiten ( 9 - 16\%), que suponen casi la mitad de todos los casos (41\%). El verbo -schreiten aparece hasta cuatro veces con el prefijo $z u-$, mientras que -gehen aparece con varias partículas, siempre una sola vez: vor-, vorbei-, her-, zu-, zurück- у zu Fuß. Aparte de -schlendern, los demás VVD aparecen de forma esporádica.

Las correspondencias entre UG y HI (alemán-español):

1) Traducciones con andar: 49 token, que se emplearon para traducir 11 VVD distintos y 4 expresiones que en el original no son estrictamente descripciones de desplazamiento. De nuevo, el más frecuente, con mucho, es -gehen (20 - $40 \%)$, y esta vez aparece -laufen (11 - 22,5\%). A continuación -wandern 
y -ziehen aparecen tres veces, -spazieren, dos veces y los demás solo una vez: -schleichen, -stapfen, -stobern, -stolpern, -trotten y -watscheln.

2) Traducciones con caminar: 11 token, que han traducido 4 VVD y una expresión. Hay tres VVD que aparecen tres veces, -gehen, -laufen y -wandern, y uno que aparece una sola vez, -irren.

3) Estos 60 token considerados corresponden a 12 VVD diferentes en alemán: los más repetidos son -gehen (23 - 38,3 \%) y -laufen (14 - 23,3\%) que suponen el $61 \%$ del total. En la mayor parte de las construcciones no hay partículas que indiquen Trayectoria. De hecho, no hay partículas que aparezcan de modo frecuente o que puedan haber determinado la traducción al español.

\subsubsection{Análisis}

De nuevo se observan analogías claras:

- Los VVD -gehen (37) y -laufen (19 son los más frecuentes; junto con -schreiten (9), suponen algo más de la mitad de las expresiones correspondientes a andar y caminar (60 de 114 - 52,6 \%). Con cierta frecuencia aparecen -wandern (6) y -schlendern (5); los demás VVD, solo ocasionalmente.

- Los 12 VVD correspondientes del texto original alemán expresan Manera; de las expresiones traducidas al alemán, solo 7 de un total de 19.

- No han aparecido casos en los que se hagan patentes dificultades de traducción debidas a la diferente combinatoria de este tipo de VVD en una y otra lenguas.

\subsubsection{Tratamiento en diccionarios bilingües}

Los datos que nos muestran los diccionarios son los siguientes: caminar: (ir) gehen / (a pie) zu Fuß gehen / (a pie) wandern (PON); gehen / wandern / reisen / (algo) etw. zurücklegen / [...] (LEO); (zu Fuß) gehen / (excursión) wandern / (niño) laufen (LAN); gehen / wandern / laufen (zu Fuß gehen) (DIC); reisen / [...] wandern / gehen / [...] schreiten / zu Fuß gehen [...] (BEO).

andar: (zu Fuß) gehen [...] (PON); gehen / laufen / wandern / [...] schreiten (LEO); gehen / (vehículo) fahren (LAN); laufen (zu Fuß gehen) / gehen (DIC); schreiten / [...] marschieren / gehen / zu Fuß gehen / fahren / zurücklegen / [...] laufen (BEO).

Para ser dos VVD sinónimos, todos los diccionarios dan equivalencias realmente distintas. 
Frente a lo que sucedía con volver y regresar, las entradas correspondientes a caminar y andar apenas se diferencian entre sí por el número de correspondencias. Llama la atención de estas que gehen aparezca en todas, pero laufen solo en la mitad y de forma nada exhaustiva, y que wandern se cite siempre como correspondencia de caminar.

\subsubsection{Contrastes}

Del cruce de los datos observados en el corpus y en los diccionarios, se desprenden varias conclusiones:

- De nuevo el peso de las correspondencias recae en unos pocos VVD: en este caso, -gehen, -laufen, -schreiten y -wandern sin partículas específicas, de los cuales solo el primero aparece con mucha frecuencia reflejado en los diccionarios.

- Como en los otros casos, los diccionarios ofrecen equivalencias adecuadas para recepción, pero no siempre para producción.

- Ofrecen también diversas «rarezas» lexicográficas: Leo y Beolingus proponen reisen como equivalente de caminar, Langenscheidt y Beolingus proponen fahren como equivalente de andar, Langenscheidt y Leo proponen zurücklegen sin ninguna indicación de que forma una construcción necesariamente transitiva equivalente a caminar-andar una distancia.

- En las traducciones de / con caminar / andar, no obstante, aparecen muchos VVD que los diccionarios nunca relacionan con ellos.

- Se encuentran, además muchas formas compuestas con partículas direccionales (entlang-, herum, vor-, vorbei-, $z u-$, etc.) que nunca se considerarían correspondencias en los diccionarios.

\section{Conclusiones}

Las conclusiones que aporta este trabajo muestran ciertas tendencias en la expresión del desplazamiento en el par de lenguas, algunos contrastes y su influencia en la presentación de esos datos en diccionarios bilingües electrónicos.

\subsection{Sobre la expresión del desplazamiento}

Con respecto a los resultados de otros trabajos contrastivos, hay una serie de hechos especialmente llamativos 
- En los tres casos estudiados encontramos que en alemán siempre hay dos o tres VVD que suponen juntos más de la mitad de las correspondencias.

- Se determinan en alemán tres grupos: hay 1) dos VVD de uso muy general (-kommen y -gehen), 2) otros menos frecuentes, asociados claramente a correspondencias concretas (volver / -kehren; salir / -treten o verlassen; andar / -laufen o -schreiten) y 3) muchos VVDM de uso muy esporádico.

- Siempre hay numerosos VVD equivalentes en alemán: volver / regresar tienen 12 equivalencias en JM y 11 en UG; salir, 12 equivalencias en JM y 18 en UG; finalmente andar / caminar, 19 en JM y 15 en UG.

- En el texto traducido del español, la expresión de Manera es más infrecuente y la variedad de VVD es menor $(2+9+10=21$ VVD) que en el texto originalmente escrito en alemán $(6+19+12=37$ VVD). Ello es más evidente en el caso de las equivalencias de salir y mucho menos en el caso de las equivalencias de andar y caminar, que ya de por sí expresan Manera.

- Finalmente, las equivalencias de los VVDT, en el caso de volver / regresar dependen de la presencia de las dos particulas zurück- $\mathrm{y}$, aunque en menor medida, wieder-. En el caso de salir, aparte de la presencia de hinaus- / heraus-, son frecuentes los complementos locativos de origen con la preposición aus. Con andar / caminar aparecen ocasionalmente diversas partículas, dependiendo de la Trayectoria expresada.

\subsection{Sobre las equivalencias halladas en los diccionarios}

Se ha sugerido que los diccionarios van dirigidos a un público mucho más ideal que real (Gelpí 2003: 319), lo que se ha vuelto más evidente con el advenimiento de internet: usuarios con distintas lenguas emplean un mismo diccionario electrónico, en ambas direcciones, con diferentes conocimientos y expectativas, tanto para recepción como para producción 7 . Así, sería excelente ofrecer a los usuarios información amplia y representativa, seleccionando la más pertinente, y ayudarles con la microestructura, presentando la información clasificada según criterios de frecuencia de uso y proximidad de significado entre unidades léxicas.

\footnotetext{
7 Los datos indicados por Domínguez (2016a: 291) muestran los mismos porcentajes de usuarios $(89,47 \%)$ que emplean con frecuencia los diccionarios bilingües ya sea para conocer el significado de una palabra ya sea para expresar el significado de una palabra en la lengua extranjera.
} 
- El análisis de los datos del corpus ofrece resultados de interés para la representación de los VVD en diccionarios bilingües: hay equivalencias claras para un número representativo de casos, como también equivalencias infrecuentes para otros casos. Ello facilita en cierto modo la función de las consultas de recepción en la dirección español-alemán, pero en absoluto las de producción, que exige muchas entradas en la dirección alemán-español y, sobre todo, criterios concretos sobre qué equivalencias ofrecer y cómo hacerlo.

- A partir del cruce de los datos del corpus y los diccionarios bilingües, el resultado claro es que se podría esperar de todos ellos más sistematicidad y exhaustividad al elaborar tanto la macroestructura como la microestructura, mayor número de equivalencias, criterios de relevancia para escogerlas $y$, tal vez, algo de información gramatical.

\subsection{Propuestas de entradas de diccionario}

Un trabajo de Fuentes (2015: 286) indica una clave esencial para lo que aquí se ejemplifica: un buen diccionario bilingüe no presenta solo equivalencias o equivalentes sino más bien las informaciones adecuadas para solventar dificultades surgidas de la anisomorfía entre las dos lenguas.

Puesto que no hay restricciones de espacio disponible, parece más adecuado utilizar para las entradas (de momento, para las relacionadas con VVD) una microestructura que se aproxime a esta:

- Una definición, breve y clara, que aporte a los no nativos una idea de su significado sin el condicionamiento de sinónimos (aproximados), a los aprendices, un refuerzo de su léxico disponible más allá del simple glosario y a los traductores, la posibilidad de hallar por sí mismos un equivalente.

- Las equivalencias más pertinentes y frecuentes, que aporten a los aprendices extranjeros sinónimos (aproximados) dentro del léxico usual, y que a los usuarios nativos les permitan escoger una equivalencia válida para traducción.

- Si es necesario, indicaciones breves de carácter sintáctico y combinatorio (vid. Cuartero 2016) que sirvan de ayuda (a veces indispensable) al usuario no nativo para construir enunciados en la lengua que no domina. 
En relación con los ejemplos que se utilizaron para ilustrar los casos tratados en los apartados previos, las entradas propuestas son las siguientes:

a) Con respecto a volver / regresar, se deben indicar las partículas del alemán que se asocian con esa Trayectoria y los VVD más frecuentes, pero también vale la pena dar una breve instrucción gramatical que prevea una buena parte de las construcciones posibles:

volver: (VD intr.) [wieder an den Ausgangspunkt gelangen=]

zurückkehren, -kommen, -gehen (en ppio. cualquier VD con la partícula zurück- explicita el significado volver/regresar); wiederkehren, -kommen; umkehren; (a casa) heimkehren, -kommen (en ppio. cualquier VD con la partícula heim- explicita el significado volver / regresar a casa).

b) Con respecto a heraus- / hinaus-, dado que no se emplean del mismo modo que los adverbios fuera / afuera del español pero, sobre todo, dado que aportan un significado constante, es preferible ofrecer una paráfrasis y no una equivalencia léxica:

hinaus-: partícula que indica que un movimiento se realiza del interior al exterior.

Una equivalencia mediante definición parafrástica tiene la ventaja de ofrecer una solución al hecho de que los diccionarios no incluyan todas las posibles formas compuestas de partícula y VD. Así, el formato digital permite crear una macroestructura sin restricciones de productividad léxica: se pueden indicar correspondencias para la partícula y el VD sin necesidad de incluir una entrada explícita para ese compuesto. Una búsqueda como hinausrennen obtendría una doble equivalencia:

hinaus-: partícula que indica que un movimiento se realiza del interior al exterior

-rennen: [desplazarse muy deprisa (a pie o en vehículo)=] ir o venir corriendo; correr.

c) Para algunas equivalencias en la dirección español-alemán, se pueden aprovechar los datos de corpus para ofrecer más homogeneidad en la presentación de equivalencias:

salir: (VD intr.) [von drinnen nach draußen kommen=]

(heraus/hinaus/raus/hervor...) -kommen, -gehen, -treten, -fahren, (en ppio. cualquier verbo de modo de desplazamiento con alguna de las partículas 
heraus/hinaus/raus/hervor - explicita el significado salir) / verlassen / ausgehen, -fahren.

d) Las equivalencias para andar y caminar podrían ser las siguientes:

andar: (VD intr.) [sich auf den Beinen gehend fortbewegen=]

(zu Fuß) gehen, laufen, schreiten; [por el campo, de excursión] wandern; [paseando] schlendern, spazieren (todos estos verbos pueden aparecer acompañados de partículas que explicitan la dirección del desplazamiento, además son compatibles con indicaciones de Origen).

andar: (VD tr.) [eine Wegstrecke gehend hinter sich lassen=] zurücklegen + distancia.

En resumen, si se enfocan no solo desde el punto de vista de la tradición lexicográfica sino desde el análisis contrastivo, los nuevos diccionarios bilingües de acceso en línea pueden permitir optimizar las herramientas básicas de aprendices, traductores y profesionales de las lenguas. Si se hacen asequibles y aprovechables para los múltiples tipos de usuarios y expectativas a las que se ha de enfrentar, los resultados estarán realmente a la altura de ese cambio de paradigma ya comenzado.

\section{Referencias bibliográficas}

BALDINGER, K. (1971): «Semasiologie und Onomasiologie im zweisprachigen Wörterbuch», en Interlinguistica: Sprachvergleich und Übersetzung. Festschrift zum 60. Geburtstag von Mario Wandruszka. En: K.-R. Bausch y H.-M. Gauger (eds.), Tübingen, Niemeyer: 384-396.

CUARTERO, J. (2015): «La expresión de desplazamiento en español y alemán. Un nuevo estudio contrastivo». En: M. Meliss y B. Pöll (eds.): Aktuellen Perspektiven der kontrastiven Sprachwissenschaft. Deutsch-Spanisch-Portugiesisch. Tübingen, Narr: 135-154.

CUARTERO, J. (2016): «Cómo se dice salir en alemán y otras serias dificultades para la lexicografía contrastiva». En: A. Castell (ed.): Sintaxis y diccionarios. La complementación en alemán y en español. Frankfurt, Lang: 161-198.

CUARTERO, J. (2017a): «Propuestas para el tratamiento de los verbos de desplazamiento del español y el alemán en diccionarios bilingües». Neuphilologische Mitteilungen 118/1: 5-32. 
CUARTERO, J. (2017b): «Estudio contrastivo de la expresión de desplazamiento en español frente al inglés y el alemán». En: M. J. Dominguez y S. Kutscher (eds.): Interacción entre gramática, didáctica y lexicografía. Berlín, De Gruyter: 325-340.

DOMÍNGUEZ, M. J. (2016a): «Dime quién eres... y te diré qué diccionario necesitas. Investigación en diccionario y su uso». En: A. Castell (ed.): Sintaxis y diccionarios. La complementación en alemán y en español. Frankfurt, Lang: 273-307.

DOMÍNGUEZ, M. J. (2016b): «Lexikographie und Wörterbuchbenutzung in einer neuen Zeit. PORTLEX als multilinguales Wörterbuch». En: J. Cuartero, J.P. Larreta y Ch. Ehlers (eds.): Querschnitt durch die deutsche Sprache aus spanischer Sicht. Berlín, Frank \& Timme: 169-186.

ENGEL, U. (1996): Deutsche Grammatik, Groos, Heidelberg.

ENGELEN, B. (2010): Schwierige sprachliche Strukturen, Frankfurt, Lang.

FUENTES, M. T. (1997): Gramática en la lexicografía bilingüe. Tübingen, Niemeyer.

FUENTES, M. T. (2015): «Representación del anisomorfismo en los diccionarios bilingües alemán-español». En: M. Meliss y B. Pöll (eds.): Aktuellen Perspektiven der kontrastiven Sprachwissenschaft. Deutsch-Spanisch-Portugiesisch. Tübingen, Narr: 285-297.

GELPÍ, C. (2003): «El estado actual de la lexicografía: los nuevos diccionarios». En: A. Medina (coord.): Lexicografía española. Barcelona, Ariel: 307-332.

HAENSCH, G. et al. (1982) La lexicografía: De la lingüística teórica a la lexicografía práctica. Madrid, Gredos.

HAENSCH, G. / OMEÑACA, C. (2004): Los diccionarios del español en el siglo XXI. Salamanca, Universidad de Salamanca.

HESS, K. (2007): Verb und Direktivum: ein Beitrag zum deutsch-spanischen und spanischdeutschen Sprachvergleich. Frankfurt, Lang.

MELISS, M. (2016): Wie viele und welche bilingualen Online-Wörterbücher brauchen wir für den DaF-Bereich? En: J. Cuartero, J.P. Larreta y Ch. Ehlers (eds.): Querschnitt durch die deutsche Sprache aus spanischer Sicht. Berlín, Frank \& Timme: 187-210.

SECO, M. (1987): Estudios de lexicografía, Madrid, Paraninfo.

TALMY, L. (2000): Toward a Cognitive Semantics: Volume II: Typology and Process in Concept Structuring. Cambridge MA, MIT Press.

WOTJAK, G. (1997): «(Her)ein/(her)aus, hinauf/herauf - hinunter/herunter. Bedenkliches und Bedenkenswertes zur Konzeptualisierung und Sememisierung 
von Fortbewegung». En: G. Wotjak (ed.): Studien zum romanisch-deutschen und innerromanischen Sprachvergleich, Frankfurt, Lang: 311-330.

DICCIONARIOS:

BEOLINGUS: diccionario en línea, disponible en https://dict.tu-chemnitz.de/de-es/

DICT.CC: diccionario en línea, disponible en https://dees.dict.cc/

LANGENSCHEIDT: diccionario en línea, disponible en

https://es.langenscheidt.com/aleman-espanol/

LEO: diccionario en línea, disponible en https://dict.leo.org/alemán-español/

PONS: diccionario en línea, disponible en https://es.pons.com/traducción

\section{FUENTES:}

ENDE, M. (1979). Die unendliche Geschichte. Stuttgart, Thienemann.

ENDE, M. (1983). La historia interminable (Traducción de M. Sáez). Madrid, Alfaguara. PÉREZ REVERTE, A. (1995): La piel del tambor. Madrid, Alfaguara.

PÉREZ REVERTE, A. (1997): Jagd auf Matutin (Traducción de C. Schmitt). Múnich, btb. 